
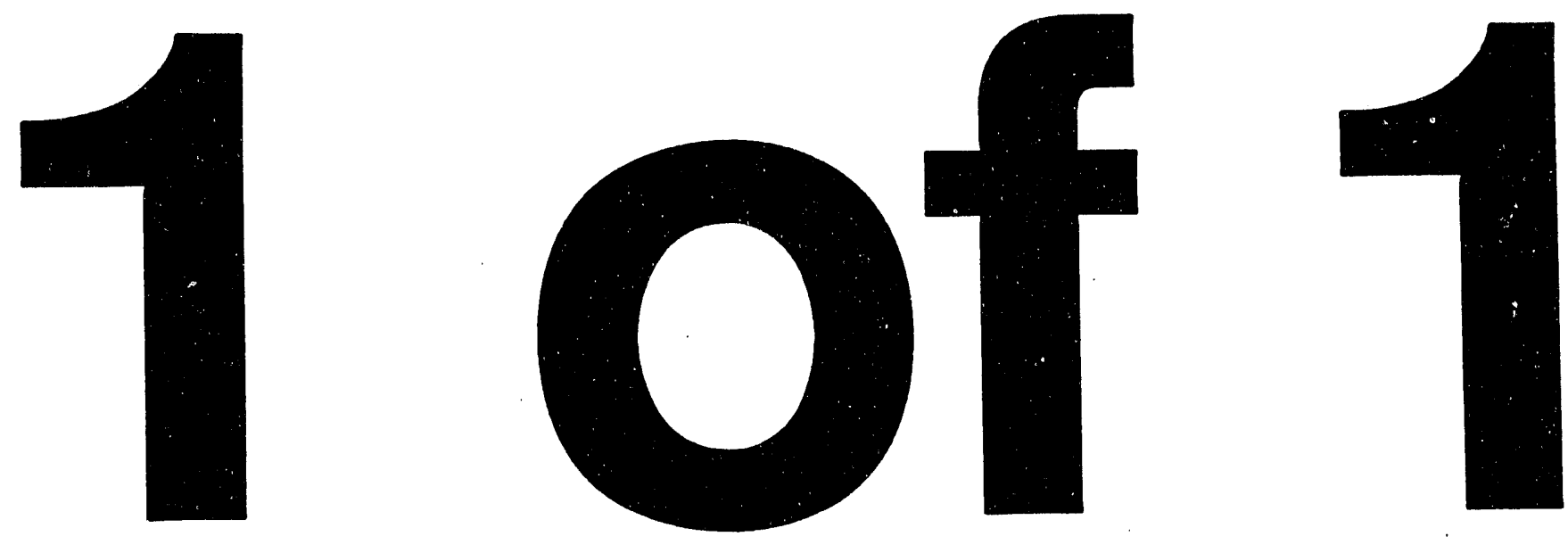


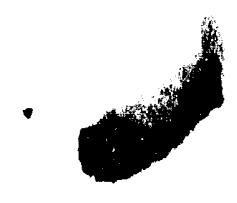

DOE/ER/40561-141 (-INT94-00-57)

\title{
Source Dimensions in Ultrarelativistic Heavy Ion Collisions
}

\author{
M. Herrmann1, G. F. Bertsch1,2 \\ 1'Institute for Nuclear Theory, University of Washington, Seattle, Washington, 98195 \\ 2Physics Department, FM-15, University of Washington, Seattle, WA 98195
}

\section{PREPARED FOR THE U.S. DEPARTMENT OF ENERGY UNDER GRANT DE-FG06-90ER40561}

This report was prepared as an account of work sponsored by the United States Government. Neither the United States nor any agency thereof, nor any of their employees, makes any warranty, express or implied, or assumes any legal liability or responsibility for the accuracy, completeness, or usefulness of any information, apparatus, product, or process disclosed, or represents that its use would not infringe privately owned rights. Reference herein to any specific commercial product, process, or service by trade name, mark, manufacturer, or otherwise, does not necessarily constitute or imply its endorsement, recommendation, or favoring by the United States Government or any agency thereof. The views and opinions of authors expressed herein do not necessarily state or reflect those of the United States Government or any agency thereof. 


\title{
Source Dimensions in Ultrarelativistic Heavy Ion Collisions
}

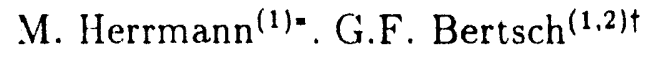 \\ (1) Institute for Nuclear Theory, HN-12, Cniversity of Washington, Seattle, WA 98195 \\ (2) Physics Department, F.M-15. University of Washington. Seattle, WA 98195 \\ (May 16, 1994) \\ Recent experiments on pion correlations, interpreted as interferometric \\ measurements of the collision zone, are compared with models that distinguish \\ a prehadronic phase and a hadronic phase. The models include prehadronic \\ longitudinal expansion, conversion to hadrons in local kinetic equilibrium, and \\ rescattering of the produced hadrons. The longitudinal radius measured in \\ collisions of $200 \mathrm{GeV} / \mathrm{u}$ sulfur nuclei on a heavy target require the existence of \\ a prehadronic phase which converts to the hadronic phase at densities around \\ $0.8 \mathrm{GeV} / \mathrm{fm}^{3}$. The transverse radii cannot be reproduced without introducing \\ more complex dynamics into the transverse expansion.
}

\section{INTRODUCTION}

Now that a second generation of nucleus-nucleus collision experiments has been completed at the CERN SPS [1], one may ask what has been learned about the dynamics of matter at high density. In particular. we want to examine the results of the interferometric pion correlation studies from the two experiments NA.35 [2] and NA44 [3]. In principle such experiments can provide quite detailed information about the space-time history of the collision [4]. Furthermore the existence of a long-lived source would show as an unambiguous signal in the measurement $[5.6]$.

\footnotetext{
"Internet address: herrmann Q̄ben.npl.washington.edu

†Internet address: bertsch@iphys.washington.edu
} 
Correlations are often analyzed to give source radii, and the measured numbers should impose some constraints on the possible dynamics. A theoretical model can be tested by modeling the collision process, and comparing model predictions with the experimental results. One difficulty in drawing firm conclusions is that there is a great deal of freedom in the construction of these models. Another problem, as we will see, is that the correlation shape is not well described by a single number, the "source size", and so it is better to compare models with the correlation function actually measured experimentally.

Our objective is to consider a broad class of models that do not go beyond established or at least widely-accepted physics, to see whether the experimental results point to new physics. This work is a continuation of a study by Welke, et al. [7], which considered earlier data and had much more detailed model assumptions. Before proceeding, we have two remarks. The first is that the two experiments, NA35 and NA44, are hardly consistent with each other in the values reported for source radii. Before any general conclusions can be drawn from the measurements, it will be necessary to resolve the disagreement. We also note that there was recently reported $[8,9]$ a study with a model, "RQMD". based on conventional physics, and good agreement was found with available data. Our conclusion is opposite: we shall find that models without explicit transverse expansion in the prehadronic phase cannot explain the body of the data.

Our paper is organized as follows. In Sec. II we discuss the physical ingredients of conventional models and develop a general parameterization of the source function. In Sec. III we discuss the Gaussian source size and its limitations. In Sec. IV we analyze what constraints data place on the models. Section V summarizes our study and gives an outlook. 


\section{MODELS AND PARAMETERIZATIONS}

We model the evolution of the system in two stages, a prehadronic, high density phase ${ }^{1}$ followed by a hadronic gas phase. Many aspects of this two-phase dynamics are subject to parameterization, but we feel we can explore the parameter space suficiently that one is testing a small set of basic assumptions about the dynamics. These assumptions are:

1) the prehadronic phase only expands longitudinally; 2) the conversion to the hadronic phase is smooth and produces hadrons with a locally thermalized kinetic distribution.

We now discuss the details of the modeling.

\section{A. High density phase}

This is the most interesting, but least understood part of the evolution. However, we do not need a great deal of information about the structure of this phase; a knowledge of the energy and momentum densities at the hadronization time should be sufficient. To be modeled are the transverse and longitudinal distributions of these quantities.

It is safe to assume that the initial transverse energy distribution follows the overlap density of the collision partners. In the detailed comparisons we will only look at central collisions of sulfur on heavy targets, so we make take the distribution to follow the transverse density of sulfur. We determined this from the charge density of Ref. [10], integrating the radial density with respect to longitudinal distance. This integral is fit very well by the following function, which we used in further numerical modeling

$$
\rho_{\perp}\left(r_{\perp}\right)=\frac{d n}{d r_{\perp}^{2}}=a\left(1+c r_{\perp}^{2}+d r_{\perp}^{4}\right) \exp \left(-r_{\perp}^{2} / b\right)
$$

The parameters have the following values: $a=1.791 \mathrm{fm}^{-3} ; b=3.79 \mathrm{fm}^{2} ; c=0.196 \mathrm{fm}^{-2}$; $d=0.021 \mathrm{fm}^{-4}$. The r.m.s. radius of ${ }^{32} \mathrm{~S}$ is $3.2 \mathrm{fm}[10]$, and the r.m.s. transverse radius is

\footnotetext{
${ }^{1}$ We use the term 'phase' just to distinguish the stages, not to imply a thermodynamic phase transition.
} 
$\sqrt{\left\langle r_{\perp}^{2}\right\rangle}=\sqrt{2 / 3} \sqrt{\left\langle r^{2}\right\rangle} \approx 2.6 \mathrm{fm}$.

We also assume that there is no transverse expansion during the prehadronic phase. Certainly there is no initial radial velocity, but it is not clear that it can be neglected completely if the prehadronic phase lasts a long time.

In the longitudinal direction, there is a rapid expansion that must be built into the models. Two distinct pictures are possible for how this happens. One kind of model [11] is based on the QCD-inspired picture of particle production by string breaking. The string is produced at a point in space-time and expands longitudinally. Particles are produced over the full initial range of longitudinal rapidities $y$, with the time and longitudinal position $(t, z)$ of the production point characterized by an equal spatial rapidity

$$
\eta=\tanh ^{-1}(z / t) \approx y
$$

Another possibility is the Landau picture, in which produced matter starts out at rest, with a small but finite longitudinal extension. Here the high longitudinal momenta arise fiom the hydrodynamic expansion. Models interpolating between these possibilities have been considered in Ref. [12] with the result that the pure Landau picture gives single-particle rapidity distributions that are too narrow. We will insist that the average rapidity of the produced particles be given by their spatial rapidity, so the distribution of produced particles will have the form ${ }^{2}$

$$
\frac{d^{6} N}{d \eta d r_{\perp}^{2} d y d m_{\perp}^{2}}=\mathcal{N} f_{\eta}(\eta) f_{y}\left(y-\eta, p_{\perp}\right) \rho_{\perp}\left(r_{\perp}\right)
$$

We have little a priori guidance on the the function $f_{\eta}$ in Eq. (1), and we consider it to be completely open to parameterization. However, the predicted single-particle distribution will depend on $f_{\eta}$, and so it is actually strongly constrained by the data. We may therefore

\footnotetext{
${ }^{2} \mathrm{By}$ assuming this form, we neglect the finite thickness of the Lorentz-contracted projectile and target nucleus. We have also tried distributions smeared out along the beam axis due to the target thickness, and found no appreciable effect on the results reported in Sec. IV.
} 
assume some simple form: we take the Gaussian,

$$
f_{\eta}=\exp \left(-\eta^{2} / 2(\Delta \eta)^{2}\right)
$$

which has as a parameter $\Delta \eta$, the r.m.s. dispersion in $\eta$.

To interpret the meaning of the parameter $\Delta \eta$ we note that for $\Delta \eta=\infty$ the initial distribution is boost invariant, i.e., corresponds to the ideal Bjorken scenario [13]. For $\Delta \eta=0$ on the other hand we get a initial distribution that is consistent with the Landau picture of a source that is fully stopped and expands starting at $\eta=0$. The parameter $\Delta \eta$ therefore interpolates between the two pictures [12].

\section{B. Formation of the hadronic phase}

We assume that the conversion to hadronic matter is controlled only by the local density as determined by the time evolution of Eq. (1). This excludes the possibility of inhomogeneities such as quark-gluon droplets. The density at which the conversion takes place, $n_{h}$, is an important parameter of the model. Because of the spatial inhomogeneity in the transverse direction, the conversion will not take place at the same proper time. Note that even with an instantaneous source in proper time, there would be a range of times in any particular reference frame. In any case, it will be useful to infer the average proper time for the formation of the hadronic gas.

Another important question at the conversion point is the composition and kinetic distribution of the newly-formed hadronic gas. One obvious possibility is a chemical and kinetic local equilibrium. Another possibility is that the distribution carries over from the distribution formed in hadron-hadron collisions.

We will assume in all our modeling that the kinetic distribution of particles is thermal with respect to the local frame defined by $\eta$. This is certainly plausible, as it will turn out that the prehadronic phase lasts a considerable time compared to estimates of the kinetic 
equilibration time. The source function $f_{y}$ will thus be taken to have a thermal form,

$$
f_{y}=\frac{m_{\perp} \cosh (y-\eta)}{\left(\exp \left(m_{\perp} \cosh (y-\eta) / T\right)-1\right)} .
$$

This has as a parameter the kinetic temperature $T$. The transverse momentum spectra from $p p$ collisions suggest a temperature of $T=130 \mathrm{MeV}$. However, we demand of our model that it fit the heavy ion data, and this will require a slightly higher value.

The chemical composition of the newly-produced hadronic matter is a much more difficult question, because there is no direct information about the composition in heavy ion collisions. For $p p$ collisions the ratio of rho mesons to final state pions has been measured [14-16], and is in the range 0.09-0.13. Note that this is significantly smaller than for the hadronic jets produced in $e^{+}-e^{-}$annihilation. Our model also includes omega mesons. The omega mesons have an important effect on the pion source at large distance [17]. Since the main difference between rho and omega mesons is their isospin, it is reasonable to suppose that they are produced as the ratio of isospin degeneracies, $N_{\rho}: N_{\omega}=3: 1$. Assuming that all pions are produced either directly or via rho and omega decay, this translates into a ratio of rho mesons to pions at hadronization time of $w_{\rho / \pi}=0.12-0.21$. Other authors have used thermal parameterizations of the particles abundances [18] which lead to a value near 0.10 for the latter ratio. We will test the sensitivity of the extracted source size to this ratio.

We do not explicitly include $\eta$-mesons in our simulation. From HELIOS data [19] we estimate that the $\eta$ 's contribute $5 \%$ to the total number of pions in the final state. Due to there very long lifetime their contribution will only reduce the intercept of the correlation function by 0.1 and will therefore have no influence on the determination of the source radii.

A further source of secondary pions is the decay of the $K^{*}$ resonance. In pp-collisions at $400 \mathrm{GeV}$ the number of produced $K^{\prime *}$ resonances was found to be similar to the number of produced $\omega$ 's [16]. As there is only one pion produced per $K^{*}$ decay we neglect this contribution.

Recently a considerable net proton number $d N_{p} / d y \approx 8$ has been reported for $\mathrm{S}+\mathrm{Ag}$ and $S+A u$ central collisions [20]. However. the number of mesons is more than an order 
of magnitude larger, and we think the presence of the baryons can be safely ignored fo observables such as the $\mathrm{HBT}$ radii.

\section{Hadronic gas}

As discussed in the previous section, the hadronic gas phase consists of pi, rho and omega mesons. These particles are allowed to propagate between interactions, to scatter pairwise, and to decay. This is a dilute gas approximation, which of course breaks down at high densities either because multiparticle interactions become important or because the mesons are no longer the appropriate degrees of freedom. In either case, we may view the parameter $n_{h}$ as determining the density at which the dilute hadronic gas picture becomes valid.

The most important (and best known) part of the elastic hadron-hadron cross-sections is the interaction among the pions. We use the momentum-dependent, isospin-averaged $\pi \pi$ cross section calculated from phase-shifts [21]. The cross sections for other mesons is not known. Given that the pi-pi cross section is around $20 \mathrm{mb}$, it is likely that the other cross sections are within the range of $10-40 \mathrm{mb}$, and we consider this as the allowable parameter range. Inelastic cross sections are more problematic, except for the well-known $\pi \pi \rightarrow \rho$ cross section. In principle, explicit inclusion of this process should lead to larger source sizes because the rho meson will propagate some distance before decaying. However, in the actual modeling it makes no appreciable change in the HBT radii whether or not this process is included. Other inelastic processes are neglected. It seems likely that pion-number changing processes have too small a rate to affect the dynamics on the time scale of the collision [22].

\section{CORRELATION FUNCTION AND RADII}

We now summarize some of the basic formulas for the interferometric analysis of currelations. First of all, the correlation function is defined as the ratio of the two-particle probability $P_{2}$ to the product of the single-particle probabilities $P_{1}$, 


$$
C\left(\overrightarrow{p_{1}}, \overrightarrow{p_{2}}\right) \equiv \frac{P_{2}\left(\overrightarrow{p_{1}}, \overrightarrow{p_{2}}\right)}{P_{1}\left(\overrightarrow{p_{1}}\right) P_{1}\left(\overrightarrow{p_{2}}\right)}
$$

It is convenient to replace the single-particle momentum variables $\overrightarrow{p_{1}}$ and $\overrightarrow{p_{2}}$ with the average momentum $\vec{Q}$ and relative momentum $\vec{q}$

$$
\begin{aligned}
& \vec{Q} \equiv\left(\overrightarrow{p_{1}}+\overrightarrow{p_{2}}\right) / 2, \\
& \vec{q} \equiv\left(\overrightarrow{p_{1}}-\overrightarrow{p_{2}}\right),
\end{aligned}
$$

and we shall use these variables as the arguments of the correlation functions below. Making certain statistical assumptions [23] about the production of particles, the correlation function can be calculated from the single-particle source function $g(x, p)$ by the formula $[24,23]$

$$
C(\vec{Q}, \vec{q}) \equiv 1+\frac{\int d^{4} x \int d^{4} y g(x, Q) g(y, Q) \cos (q(x-y))}{\int d^{4} x g(x, Q+q / 2) \int d^{4} y g(y, Q-q / 2)} .
$$

Here the variables $Q$ and $q$ are four-vectors with time-like components given by $Q_{0}=$ $\frac{1}{2}(\omega(\vec{Q}+\vec{q} / 2)+\omega(\vec{Q}-\vec{q} / 2))$ and $q_{0}=\omega(\vec{Q}+\vec{q} / 2)-\omega(\vec{Q}-\vec{q} / 2)$. Note that the fourmomentum argument of the source function in the numerator is off-shell, i.e. $Q^{2} \neq m_{\pi}^{2}$.

One often discusses correlations for fixed $\vec{Q}$, in which case the $\vec{q}$ dependence leaves three degrees of freedom in the correlation function. We single out three orthogonal axes to vary $\vec{q}$, working in a frame in which the component of $\vec{Q}$ along the beam axis vanishes. The three components of the correlation function are:

1. the longitudinal correlation function $C_{l}$ with $\vec{q}$ parallel to the beam axis, $\vec{q} \| \hat{z}$;

2. the outward correlation function $C_{o}$ is defined with $\vec{q}$ tranverse to the beam axis and along $\vec{Q}, \vec{q} \| \vec{Q}$

3. the sideward correlation function $C_{s}$ with $\vec{q} \perp \vec{Q}$ and $\vec{q} \perp \hat{z}$.

It is convenient to characterize each of these correlation functions by a single number, the source radius. This is usually taken as the r.m.s. extension of the source along some axis, but let us start with an operational definition in terms of correlation functions themselves. We define the radii by

$$
R_{i}^{2} \equiv-\vec{\nabla}_{q_{i}}^{2} C(\vec{Q}, \vec{q})_{q=0}
$$


where $i$ labels the three possible directions. This is double the mean square source size defined by $\left\langle r_{i}^{2}\right\rangle$, if the single-particle source has no momentum dependence. In general, the relation is [23]

$$
R_{i}^{2}(Q)=2\left\langle\left(x_{i}-\iota_{i}(Q) t\right)^{2}\right\rangle-2\left\langle x_{i}-\iota_{i}(Q) t\right\rangle^{2}+\frac{1}{2}\left(\left\langle\frac{1}{g} \partial_{Q_{1}}^{2} g\right\rangle-\left\langle\frac{1}{g} \partial_{Q_{1}} g\right\rangle^{2}\right)
$$

Here the expectation value is with respect to the space-time distribution in the source function $g(x,(\omega(Q), \vec{Q}))$,

$$
\langle f\rangle=\frac{\int d^{4} x f \tilde{g}(x, Q)}{\int d^{4} x \tilde{g}(x, Q)}
$$

and $\partial_{Q_{1}}$ is a partial derivative with respect to the spatial $Q_{i}$ dependence in the source function. If the particle distribution is in local thermal equilibrium, then $g$ is a function of $x$ and $\omega(Q)$ alone. Therefore we expect that effects of the momentum dependence of the single-particle source which appear in the last two terms will be rather small.

One point of principle is worth emphasizing at this point. In a classical simulation we can only get information about the source function for on-shell momenta. It is common to calculate a classical, on-shell correlation function according to

$$
C_{c l}(\vec{Q}, \vec{q}) \equiv 1+\frac{\int d^{4} x \int d^{4} y g(x, Q+q / 2) g(y, Q-q / 2) \cos (q(x-y))}{\int d^{4} x g(x, Q+q / 2) \int d^{4} y g(y, Q-q / 2)} .
$$

With this definition of the correlation function, the source size evaluated from Eq. (5) has only the first two terms in Eq. (6), i.e.,

$$
R_{c l, i}^{2}(Q)=2\left\langle\left(x_{i}-v_{i}(Q) t\right)^{2}\right\rangle-2\left\langle x_{i}-v_{i}(Q) t\right\rangle^{2}
$$

This is equivalent to the definition as an r.m.s. source size. As discussed above. we expect the effect of the additional terms associated with the quantum formula to be small. but this can only be quantified with a quantum mechanical calculation that contains the necessary off-shell information.

In the sequel we will evaluate Eq. (8) in two ways. The first is to replace $g(x, Q)$ by a sum over $\delta$-functions that represents the positions and momenta of the pions at their last 
interaction as it is done, e.g., in Ref. [8]. The other way is to fit a smooth function to the result of our simulation. The second choice has the advantages that we can demonstrate the dependence on various parameters explicitly. In the models the source function is symmetric about the beam axis, and we may take the spatial variables to be the longitudinal position $z$, the transverse distance from the center $r_{\perp}$, and the angle in the transverse plane between $\overrightarrow{r_{\perp}}$ and $\vec{Q}, \phi$. The expressions for the three source radii are then

$$
\begin{gathered}
R_{s}^{2}=2\left\langle r_{\perp}^{2} \sin ^{2} \phi\right\rangle, \\
R_{o}^{2}=2\left[\left\langle\left(r_{\perp} \cos \phi-v(Q) t\right)^{2}\right\rangle-\left(\left\langle r_{\perp} \cos \phi-v(Q) t\right\rangle\right)^{2}\right], \\
R_{l}^{2}=2\left\{\left\langle z^{2}\right\rangle-\langle z\rangle^{2}\right\} .
\end{gathered}
$$

We first want to remark that the correlation in $\phi$ will reduce the effective size of the source. If the source were isotropic (no $\phi$ dependence), $\left\langle\sin ^{2} \phi\right\rangle=1 / 2$ and the sidewards radius is just the r.m.s. transverse radius. If the source function contains a strong correlation between $\hat{r}_{\perp}$ and $\vec{Q}$ the sideward radius will be smaller than the actual transverse extension. This effect has been discussed for exploding sources or collective transverse flow [24].

For the outward radius, the expression with a factorizable source function is

$$
\begin{aligned}
R_{o}^{2}= & 2\left[\left\{\left\langle r_{\perp}^{2}\right\rangle\left\langle\cos ^{2} \phi\right\rangle-\left\langle r_{\perp}\right\rangle^{2}\langle\cos \phi\rangle^{2}\right\}\right. \\
& \left.+v(Q)^{2}\left\{\left\langle t^{2}\right\rangle-\langle t\rangle^{2}\right\}\right] .
\end{aligned}
$$

This effect of the $\phi$ correlation can have either sign here. but a reduction is expected. For a very close correlation between $\hat{r}_{\perp}$ and $\vec{Q}$, the first term reduces to a finite value. the radial dispersion of source points, while the second term adds a dispersion in time. In this limit the sidewards source size goes to zero, so one certainly expects a larger outward radius than sidewards radius.

The longitudinal radius measures most directly the freeze-out time of the system. For 
thermal emission of a boost invariant system at a freeze-out proper time $\tau_{f}$ (see footnote ${ }^{3}$ ), the longitudinal radius for particles at rapidity $y=0$ is given by

$$
R_{l}^{2}=2 \frac{\tau_{f}^{2} T}{m_{\perp}} \frac{\kappa_{2}\left(m_{\perp} / T\right)}{K_{1}\left(m_{\perp} / T\right)} .
$$

In the limit $m_{\perp} \gg T$ this reduces to the formula [25] $R_{l}^{2}=2 \tau_{f}^{2} T / m_{\perp}$ which is sometimes used to deduce hadronization times (e.g., Ref. [2]). For realistic values of the temperature and transverse momentum the factor $m_{\perp} / T$ is of the order of one and Eq. (14) should be used.

\section{NUMERICAL SIMULATIONS}

For the numerical studies we used a program made from the elements of Boggs ' cascade program [26]. The first part of the program initializes the distribution of hadrons. which will materialize at different times. The parameterized density distribution, Eq. (1), is sampled with the help of the Metropolis method to give a space-time distribution of hadrons having the desired number of hadrons and a composition specified by the parameter $w_{\rho / \pi}$. The materialization time of each hadron is determined as the time when the local density from Eq. (1) falls below the critical value $n_{h}$. The hadrons are also given an initial momentum, obtained by sampling the Maxwell-Boltzmann distribution in a frame boosted to rapidity $\eta$. The remainder of the program is a loop stepping through time. In each time interval, the program goes through the following tasks:

-determine which hadrons have materialized:

-propagate in space according to the hadron's velocity;

-collide hadrons pairwise according to the assumed cross sections and their impact

\footnotetext{
${ }^{3}$ The freeze-out time $\tau_{f}$ is the time of the last interaction of a particle. This is not to be confused with the hadronization time $\tau_{h}$ with is the time when a hadron emerges from the prehadronic phase
} 
parameters ${ }^{4}$

-convert omega and rho resonances to pions according to their time-dilated lifetimes.

Each run of the program creates a file of the final pions containing their momenta and the space-time coordinates of their last interaction point. For the studies reported here, we typically average over 100 runs.

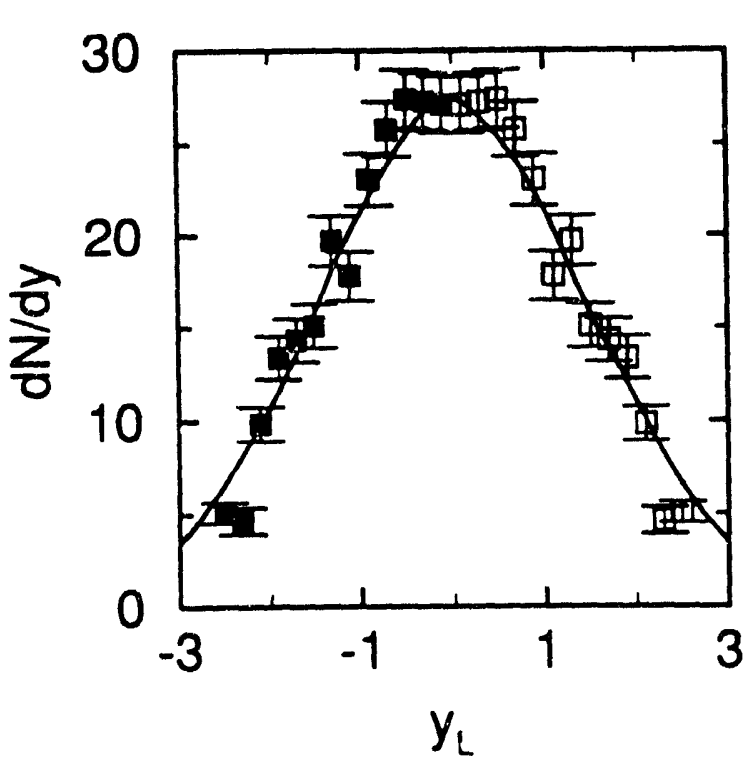

(a)

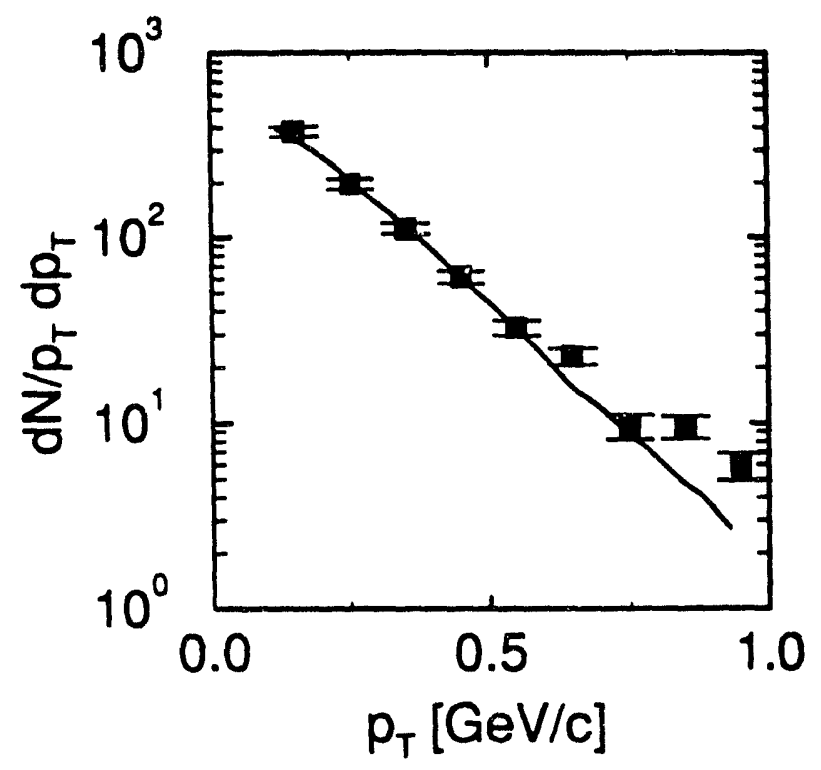

(b)

FIG. 1. The single-particle distributions $d N / d y$ and $d N / p_{T} d p_{T}$ from [20] (full squares) for $S+S$ and our simulation (full line) using the base set of parameters. We constructed the open squares in (a) by assuming symmetry around C.M rapidity $y=3$. The data in (b) are for the laboratory rapidity window $2<y_{L}<3$. The normalization of our results in both figures is arbitrary.

As formulated, the model contains as parameters the extension of the collision zone

${ }^{4}$ This is done using the method of $[27]$. This contains as a parameter $b_{0}$, the maximum impact parameter allowed for a collision. The extracted radii are insensitive to $b_{0}$ for values in the physical range, $b_{0} \sim 2 \mathrm{fm}$, but change by a few tenths of a fermi for much larger values. 
in spatial rapidity $\Delta \eta$, the density at the transition to the hadronic gas $n_{h}$, the kinetic temperature at the transition $T$, the proportion of vector mesons at the transition and scattering cross sections involving vector mesons. We first note that a number of these parameters are strongly constrained by the empirical single-particle spectra. In particular, the final state rapidity distribution depends strongly on $\Delta \eta$, weakly on $T$, and hardly at all on any of the other parameters. Correspondingly, the average transverse momentum depends almost exclusively on $T$. We may therefore consider these parameters to be fixed by the data. Single particle spectra for $\mathrm{S}+\mathrm{Au}$ or $\mathrm{S}+\mathrm{Pb}$ are not available yet, but NA35 results for $\mathrm{S}+\mathrm{S}$ at $200 \mathrm{GeV} / \mathrm{u}$ have been published [20]. In the remaining calculations we take values of $\Delta \eta=1.2$ and $T=150 \mathrm{MeV}$ to fit the shape of the distributions from this measurement. The quality of the fit is shown in Fig. 1, where we have assumed values of the other parameters given in Tab. I.

TABLE I. Source radii for $\mathrm{S}+$ heavy target. Parameter values are: $n_{h}=1.0 \mathrm{pions} / \mathrm{fm}^{3}$, $w_{\rho / \pi}=0.16, T=150 \mathrm{MeV} . \Delta \eta=1.2$.

\begin{tabular}{|c|c|c|c|c|}
\hline \hline & $\begin{array}{c}R_{l} \\
(\mathrm{fm})\end{array}$ & $\begin{array}{c}R_{0} \\
(\mathrm{fm})\end{array}$ & $\begin{array}{c}R_{s} \\
(\mathrm{fm})\end{array}$ & $\begin{array}{c}\lambda \\
\text { see footnote }\end{array}$ \\
\hline Eq. (5) & 12. & 15. & 7.5 & 1 \\
$\tau<20 \mathrm{fm} / \mathrm{c}$ & 4.3 & 4.5 & 2.9 & 1 \\
fit to Eq. (15) & 4.0 & 3.6 & 3.2 & 0.82 \\
as above. $\tau<20 \mathrm{fm} / \mathrm{c}$ & 4.0 & 3.6 & 3.2 & 0.94 \\
\hline NA35 & 4 & $4+$ & $4+$ & $5.2-6.2$ \\
NA44 & $6.2-6.9$ & $5.1-5.8$ & $0.6-0.7$ \\
\hline \hline
\end{tabular}

${ }^{5}$ Our simulation does not include the effect of $\eta$ meson decays on the correlation function, which will only lead to a change of the intercept. Therefore $\lambda$ is listed for the sake of completeness only. 
We want to emphasize again that the single-particle observables are very insensitive to the other parameters once the temperature and the space rapidity spread are fixed.

We determined the absolute normalization from the number of negative particles at midrapidity for $\mathrm{S}+\mathrm{Au}$ as measured by NA35 [28]. The measured number, $d N / d y \approx 65$, is reproduced taking the total number of pions in the final state to be 700 .

The most important remaining parameter is the hadronization density $n_{h}$. For a first comparison with data we will use the only recent publication of a correlation function, the data of NA44 in Ref. [29]. This data is shown in Fig. 2.

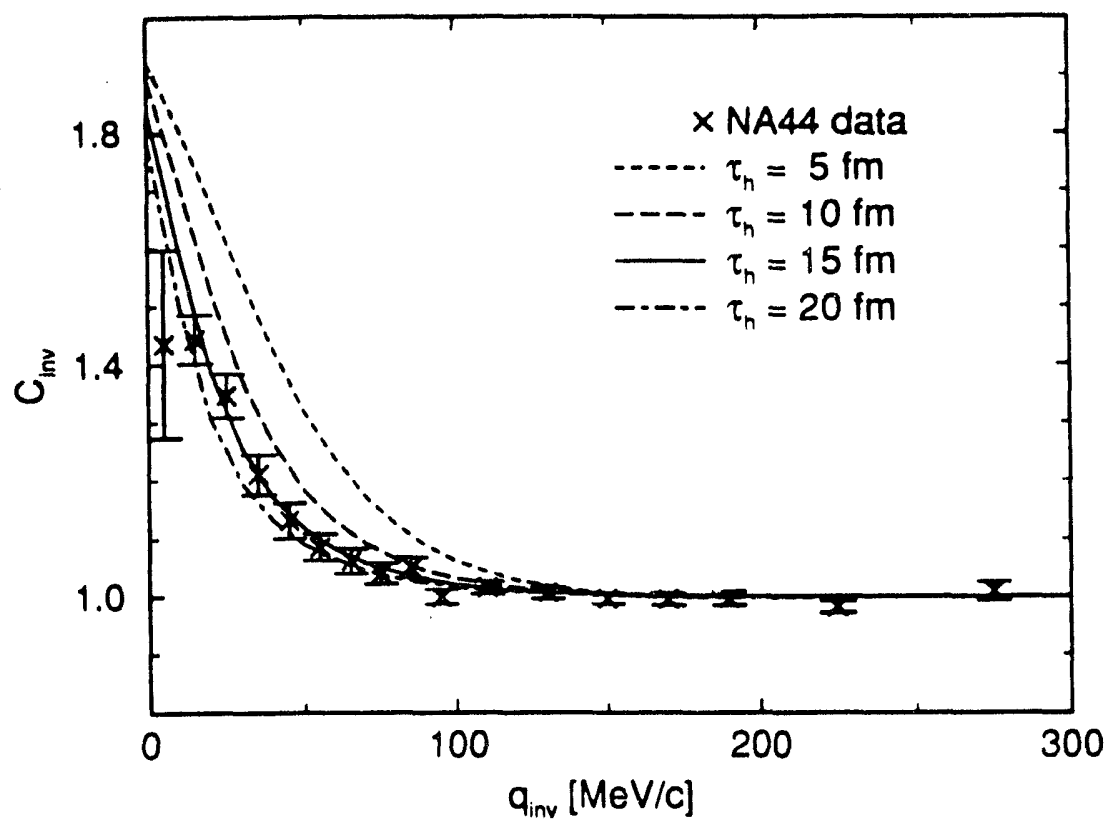

FIG. 2. Correlation function as a function of the invariant momentum for different values of the hadronization time $\tau_{h}$. We used our base set of parameters except for the vector meson to pion ratio that was chosen to be $w_{\rho / \pi}=0.10$. The crosses show the NA44 results from Ref. [29].

The correlation is plotted as a function of the invariant momentum $q_{i n v}=\sqrt{-q^{2}}$. In this comparison, we do not use $n_{h}$ directly as a parameter, instead assuming that the hadronization occurs at a definite proper time $\tau_{h}$. We show in Fig. 2 the model results for various $\tau_{h}$, and taking the proportion of heavy mesons at 0.10 . We applied cuts to our pion output files 
that are similar to the NA44 acceptance. It may be seen from the curves that agreement with data can only be obtained for very long proper times, of the order of $15 \mathrm{fm} / \mathrm{c}$. Using Eq. (1) we find that the highest density at this time is $0.2 \mathrm{pions} / \mathrm{fm}^{3}$, a number which is so low that the gas would be noninteracting at that point. Clearly, drastic assumptions would have to be made for the dynamics of the prehadronic phase to obtain conversion at such extended times.

We now turn to the three-dimensional analyses of the source geometry, which should in principle provide more detailed information. Here we shall see a less dramatic inconsistency, but one which seems difficult to overcome without introducing new dynamics.

The usual analyses of source geornetry assume Gaussian source shapes, which actually do not describe the source shape well. For that reason the extracted radii bear little relation to the definition, Eq. (5). We illustrate this with a typical calculation, with parameters and results shown in Tab. I. This parameter set is fit to the inclusive spectra as well as the Gaussian longitudinal radius from NA35 [2]. The favored hadronization density, $n_{h}=1.0$ pions $/ \mathrm{fm}^{3}$, corresponds to an average hadronization proper time, $\tau_{h}=2.3 \mathrm{fm}$ with a standard deviation $\sigma=1.5 \mathrm{fm}$. The mean hadronization time is large compared to theoretical estimates of the equilibration time of the prehadronic phase $[30,31]$, supporting our assumption of local thermal equilibrium. The spread in proper hadronization time is too big to allow for the simplification of a hadronization at a particular proper time instead of the more physical assumption of a hadronization density. The first row in Tab. I shows the extracted source radii from the operational definition, Eq. (5). This equation can only be used directly if we know the correlation function with a high resolution for small relative momenta. To circumvent the problem with low statistics we fit a smooth function to the distribution of last interaction points from the simulation. This is shown in detail in App. A.

These numbers are quite large due to the decay of the omega meson at large distances from the reaction zone. Removing particles that decay after $\tau=20 \mathrm{fm} / \mathrm{c}$ gives the numbers in the second row. We see that they are considerably smaller. Next we fit to a Gaussian parameterization of the correlation function, 


$$
C_{f}=1+\lambda \exp \left(-\frac{1}{2}\left(q_{l}^{2} R_{l}^{2}+q_{s}^{2} R_{s}^{2}+q_{o}^{2} R_{o}^{2}\right)\right)
$$

Numerically, we make the fit following the same procedure used by the experimentalists. Namely, we bin the three-dimensional correlation function (bin width $10 \mathrm{MeV} / \mathrm{c}$ ), and make a four-parameter least squares fit. We take pions in the interval $100 \mathrm{MeV} / \mathrm{c}<p_{\perp}<300$ $\mathrm{MeV} / \mathrm{c}$ and $|y|<1$. The results are shown in the third row. These radii are within $1 \mathrm{fm}$ of the true source radii with late decays excluded. The table also shows the Gaussian fit with the late-decaying particles excluded. This gives practically identical radii, although the value of $\lambda$ is somewhat changed. Thus, the 3D Gaussian fit is insensitive to the contribution of the long-lived resonances. One last point to note is that the difference between outward and sideward source radii is rather suppressed in the 3D Gaussian fit.

The experimental results for reactions at $200 \mathrm{GeV} / \mathrm{u}$ for various projectile-target systems have been published in conference proceedings $[2,3,32]$. There are no high precision data from two different collaborations for the same target-projectile combination, but the $\mathrm{S}+\mathrm{Pb}$ system studied by NA44 should be very similar to the S+Au system studied by NA35. However, the numbers for the source radii quoted by these groups are quite different. In Tab. I, we have extracted numbers for the the NA35 experiment from their Figs. 2,3 and 4 in [2], estimating an average size for the momentum range $100 \mathrm{MeV} / \mathrm{c}<p_{\perp}<300 \mathrm{MeV} / \mathrm{c}$. For the NA44 numbers, we quote the $\pi^{+}$and $\pi^{-}$radii for the $\mathrm{S}+\mathrm{Pb}$ system in Tab. II of Ref. $[3]^{6}$.

The baseline parameter set for our model is chosen to fit the NA.35 longitudinal source radius $^{7}$ as well as the single-particle distribution. Note that neither the outward nor the

${ }^{6}$ The NA44 numbers are changed by a factor of $\sqrt{2}$ to correspond to the definition Eq. (5).

TThe use of Eq. (14) to translate this radius into a freeze-out time leads to $\tau_{f}=2.5 \mathrm{fm} / \mathrm{c}$, which is much smaller than the actual average last interaction time. The Eq. (14) cannot be used because it was derived assuming boost invariance. Furthermore is the temperature much lower when the particles freeze-out than when they hadronize. 
sidewards radii are fit here.

We next examine the effects of varying the parameters. A number of cases are shown in Tab. II, compared with the results of the baseline parameter set. The second line shows the effect of increasing the number of primordial vector mesons in the hadronic phase by $25 \%$. There is virtually no effect on the radii. but the normalization $\lambda$ is lowered. We nex $\hat{\imath}$ examine the effect of a different cross section in the hadronic phase. The baseline parameters were modified by doubling all the hadronic cross sections for the entries in the third line of the table. Again, the effect on the radii is very slight. Finally, the fourth and fifth lines show the effect of changing the hadronization density by $+50 \%$ and $-60 \%$, respectively. It may be seen that this has a strong effect on the longitudinal radius. Thus, the longitudinal radius gives a good measure of the hadronization density. There is also a significant effect on the outward radius. This is because the outward radius depends on the time distribution of the emission of hadrons, which is broader when the conversion is a lower density. The sidewards radius is hardly affected by the hadronization density, but this is a consequence of our basic assumption that there is no sidewards expansion in the prehadronic phase.

TABLE II. Sensitivity of source radii to model parameters.

\begin{tabular}{|c|r|r|r|r|}
\hline \hline & $R_{l}$ & $R_{o}$ & $R_{s}$ & $\lambda$ \\
& $(\mathrm{fm})$ & $(\mathrm{fm})$ & $(\mathrm{fm})$ & \\
\hline base & 4.0 & 3.6 & 3.2 & 0.82 \\
$w_{\rho / \pi}=0.21$ & 4.0 & 3.7 & 3.2 & 0.78 \\
$\sigma \times 2$ & 4.0 & 3.6 & 3.4 & 0.88 \\
$n_{h}=1.50$ & 3.2 & 3.2 & 3.4 & 0.86 \\
$n_{h}=0.40$ & 8.2 & 3.0 & 5.6 & 0.77 \\
\hline \hline
\end{tabular}


The parameter changes we have discussed do not affect the one-particle observables significantly. The two remaining parameters, $T$ and $\Delta \eta$, cannot be changed without disturbing the agreement there.

\section{A. Transverse Momentum}

Recently NA35 has measured the dependence of the source size on transverse momentum [2], finding that the size shrinks as the transverse momentum is increased. This effect was predicted by Pratt [5] and is due to the collective flow, that originates in our model in the rescattering of the hadronized pions. Without this rescattering, our model would have no correlation between position and momentum and the source size would reflect the spatial extension of the entire source. The correlation between transverse momentum and position must vanish as the momentum goes to zero, so the low-momentum source size is not affected by this correlation. On the other hand, at finite momentum the correlation leads to a reduction of the measured transverse size of the system. In Fig. 3 (a) we compare the results from our simulation to the ones obtained by NA35 [2]. As before our results turn out to be much smaller than the experimental findings. However, the momentum dependence of the size agrees with the trend of the data. 


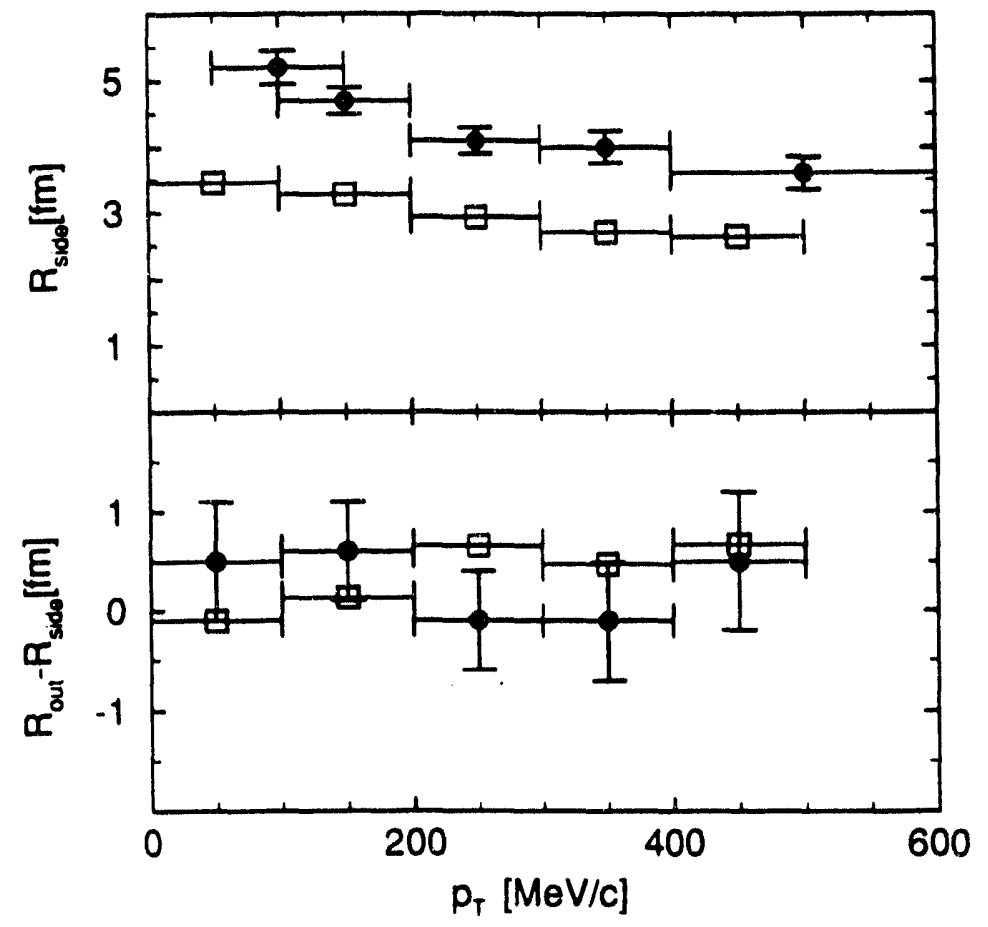

(a)

(b)

FIG. 3. The sideward radius (a) and the difference between sideward and outward radius (b) as a function of the transverse momentum. The dots are NA35 data [2] for a laboratory rapidity $3.6<y_{L}<4.6$. Our results (squares) are obtained by a 3D-Gaussian fit using the pions with a CM rapidity $0.6<y<1.6$.

In Fig. 3 (b) we show the difference between outward and sideward radius as a function of the transverse momentum. Here the experimental results [2] and the results from our simulation are essentially compatible with the difference being zero. Analogous to the results summarized in Tab. I this is, at least in our model, an artifact of the 3-D Gaussian fitting. Therefore it is probably safe to conclude that there is no very long-lived source. However, one is not forced to conclude that the particles are emitted at the same time. either. 


\section{CONCLUSIONS}

In our conclusions, we first emphasize that the source radii one extracts from pi-pi correlations depend very much on how the analysis is made. A proper definition of the radius, such as in Eq. (5), gives large radii with substantial differences in the three directions. These actual radii have a large component due to the omega meson decay, however, and they could only be measured by having momentum accuracies to much smaller than 10 $\mathrm{MeV} / \mathrm{c}$. The usual technique for extracting source radii, fitting a 3-D Gaussian as in Eq. (15), turns out to be insensitive to the resonance decays. However, there is also a much reduced discrimination between the different directions. In particular the actual outward radius is twice as large as the sideward one, but this is reduced to a $10 \%$ difference in the 3-D Gaussian fit.

We have shown that a purely hadronic model, consisting of light mesons interacting by two-particle scattering is inconsistent with the measured longitudinal radii.

Quantitatively we find that the hadronic gas picture needs to be replaced by other dynamics when the energy density is higher than about $0.8 \mathrm{GeV} / \mathrm{fm}^{3}$. The local temperature at the conversion point is constrained by the transverse momentum spectrum to be about $150 \mathrm{MeV}$. Theoretically, one no longer expects a first-order phase transition between the hadronic phase and a high-temperature quark-gluon plasma phase [33], as was first suggested. However, a remnant of that transition may persist producing a large increase of energy and entropy density over a small interval of temperature. Estimates for this temperature are in the range of $150 \mathrm{MeV}$ [34]. The energy density of a gas of gluons and quarks of two flavors is of the order of $1 \mathrm{GeV} / \mathrm{fm}^{3}$ at this temperature. Just below the transition temperature, the energy density would be that of a gas of pions and the light vector mesons, which is $0.1 \mathrm{GeV} / \mathrm{fm}^{3}$ for $T=150 \mathrm{MeV}$. By fitting the density of conversion to the experimental longitudinal radius, we are led to a hadronic gas that is far out of chemical equilibrium. Further work is necessary before conclusions can be drawn about the nature of this transition. However, it is intriguing that the energy density of a quark-gluon plasma is 
so close to the results of the model.

We also found that the predicted sidewards and outward radii were consistently small compared to experiment. This has also been found [35] for the source size predicted in the VENUS model by Werner [36]. In this model, the prehadronic phase is described by strings which decay into prehadronic clusters. Like in our models, there is no transverse dynamics in the prehadronic phase.

The small predicted sidewards radius is particularly difficult to reconcile, because the obvious remedy, an adiabatic transverse expansion, appears to have little effect on this observable $[37,38]$. Thus a transverse expansion with some nontrivial dynamics seems to be required. This would be indirect evidence of the pressure in that phase; if there were a phase transition the pressure should be quite low compared to the energy density. Any transverse expansion in the prehadronic phase would lower the required density at the conversion point. Thus our number $0.8 \mathrm{GeV} / \mathrm{fm}^{3}$ should be considered an upper limit. More work obviously needs to be done to explore the bounds on the equation of state provided by the data.

Finally we mention that the more inclusive correlation observable, given by the invariant momentum distribution, requires an unphysically large hadronization time, because the average size must be fit by adjusting only one of the three independent dimensions.

\section{ACKNOWLEDGMENTS}

We would like to thank J. Cramer, D. Ferenc, T. Humanic, B. Jacak, R. Morse, G. Roland, R. Stock, L. Teitelbaum and L. Y. Yan for helpful conversations about the experimental measurements. We would also like to thank P. Danielewicz, G. Welke and H. Schulz for discussions leading to this study. This work has been supported in parts by the Department of Energy under (irant DE-FG06-90ER40561 (G.B.. M.H.), and by the Alexander von Humboldt-Stiftung (Feodor-Lynen Program) (M.H.). 


\section{APPENDIX A: SOURCE PARAMETERIZATION}

We display in this appendix a parameterization of single-pion source function obtained with our baseline model. This parameterization is useful for determining the actual source radii from Eq. (5). It is also useful to see the influence of the long-lived resonances, which appear in the parameterization as an exponentially decaying cornponent to the source distribution.

The distribution of the emission points of the pions can be written as a function of proper time $\tau$, space rapidity $\eta$, the transverse distance to the beam axis $r_{\perp}$ and the angle between the transverse component of the position and the transverse component of the momentum $\phi \equiv L\left(\overrightarrow{p_{\perp}}, \overrightarrow{r_{L}}\right)$. These are still too many variable to make a general fit, therefore we assume that we can separate the emission into several intervals in proper time. For each of these intervals we assume, that the emission function can be factorized in the other variables, i.e.,

$$
g\left(\tau, \eta, r_{\perp}, \phi\right)=g_{i}^{\tau}(\tau) g_{i}^{\eta}(\eta) g_{i}^{r}\left(r_{\perp}\right) g_{i}^{\phi}(\phi), \quad r_{i}<\tau<\tau_{i+1}
$$

In Fig. 4 we show the proper time distribution of the pion emission, considering 100 runs taking pions with rapidity $|y|<1$ and transverse momentum in the range $100 \mathrm{MeV} / \mathrm{c}<p_{\perp}<$ $300 \mathrm{MeV} / \mathrm{c}$. This distribution can be conveniently separated into three intervals:

1. $0 \mathrm{fm} / \mathrm{c}<\tau<10 \mathrm{fm} / \mathrm{c}, 2.5 \mathrm{fm} / \mathrm{c}<\tau<10 . \mathrm{fm} / \mathrm{c}$ and $3.10 \mathrm{fm} / \mathrm{c}<\tau$. The boundaries of these regions are indicated by arrows in the figure. The distribution is well fit by a Gaussian in the first interval and exponentials in the later intervals, as shown by the lines in Fig. 4. The $r_{\perp}$ distribution is fit by Gaussians in the first and second interval and by an exponential in the third one. We also found that the $\eta$ distribution is reasonably fit by Gaussians. To determine the $\eta$ distribution we transformed each pion into its rest system. 


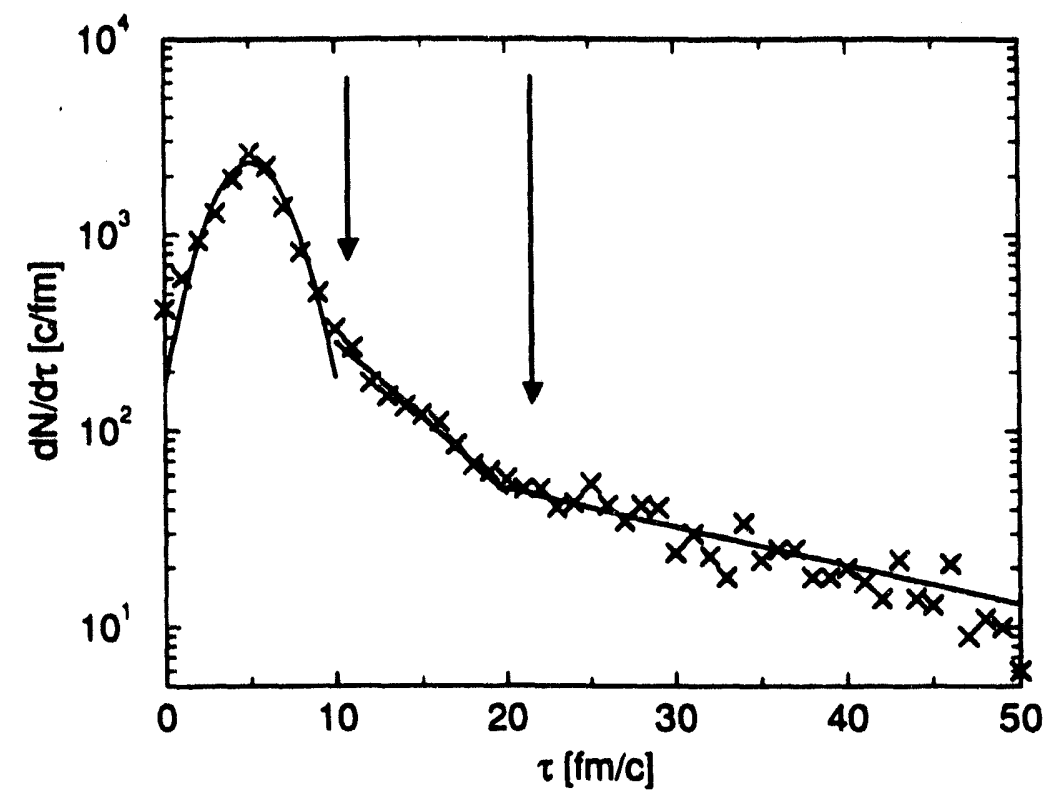

FIG. 4. The number of pions emitted per unit proper time interval as a function of proper time. The arrows indicate the boundaries of the intervals we chose for our parameterization. The full lines show our parameterization.

For the $\phi$ distribution, we need information about the first two coefficients of a Fourier expansion to calculate the outward and sidewards radii. We thus use a parameterization of the form

$$
g^{\phi}=1+b \cos (\phi)+c \cos (2 \phi)
$$

In Fig. 5 we show how well this parameterization compares with the output of the simulation program. Note that the correlation is quite strong between transverse radius and momentum. 


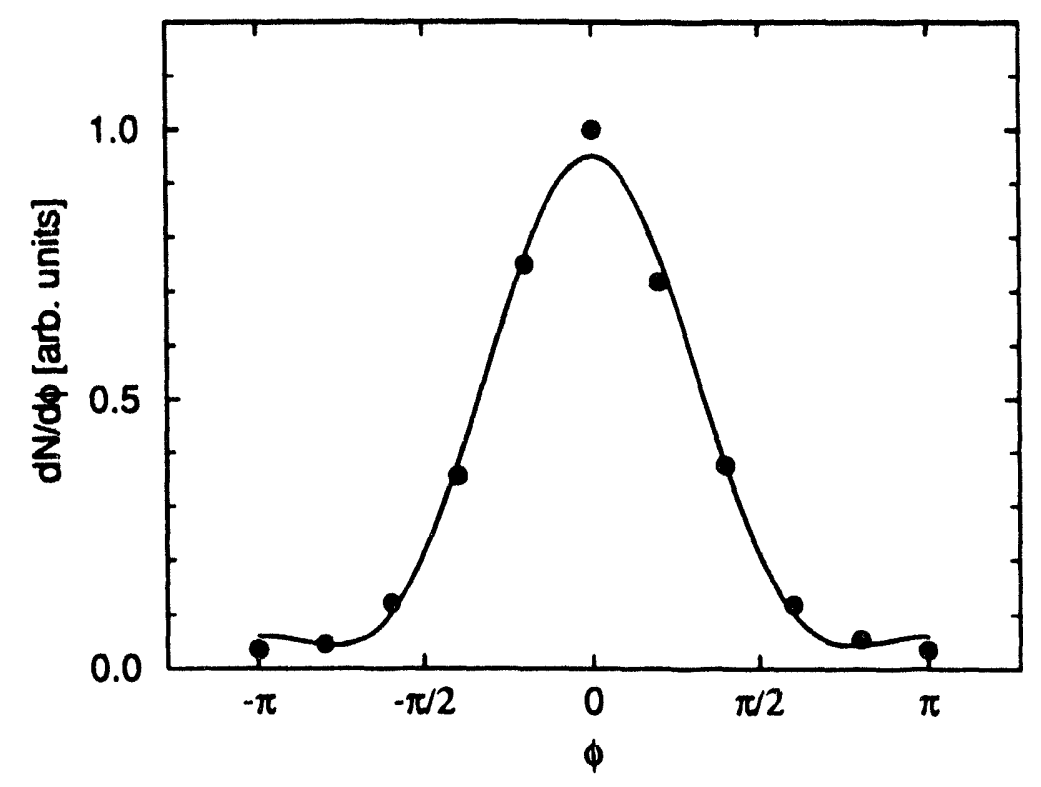

FIG. 5. Correlation between position and momentum of emitted pions, for the group emitted before $10 \mathrm{fm} / \mathrm{c}$. The dots show the probability distribution $g_{1}^{\phi}$ from the model with the base parameter set, and the line shows the fit with Eq. (A2).

The parameterization of the dependence on proper time and the other variables is summarized in Tab. III.

In Fig. 6 we show the results for the outward and sideward correlation function. Thereby we assumed all pion pairs to to have an average momentum of $200 \mathrm{MeV} / \mathrm{c}$. The crosses are obtained by using the results from our simulation directly, i.e., replacing the integral over the source function by a sum over delta functions. The dashed line shows the appropriate component of the three-dimensional Gaussian fit, with the parameters as given in Tab. I. The full line is the result using the parameterization of the source function. Note that the sum over delta functions and the Gaussian fit lead to a "coherence" parameter $\lambda<1$ whereas the correlation function extracted from the parameterization goes to 2 for small $q$. The deviation of $\lambda$ from one in the former case is entirely due to the finite binning and the limitations of a Gaussian fit, respectively. For relative momenta $q \geq 100 \mathrm{MeV} / \mathrm{c}$ our parameterization gets 
unrealistic as we have assumed that the position-momentum correlation is independent of $r_{\perp}$. This assumption is justified for the bulk part of the particles. For particles that are emitted at $r_{\perp}$ small the emission has to be isotropic and therefore $g^{\phi}$ had to go to 1 in this limit. For the extraction of the radii that are determined by the low $q$ behavior this has no consequences.

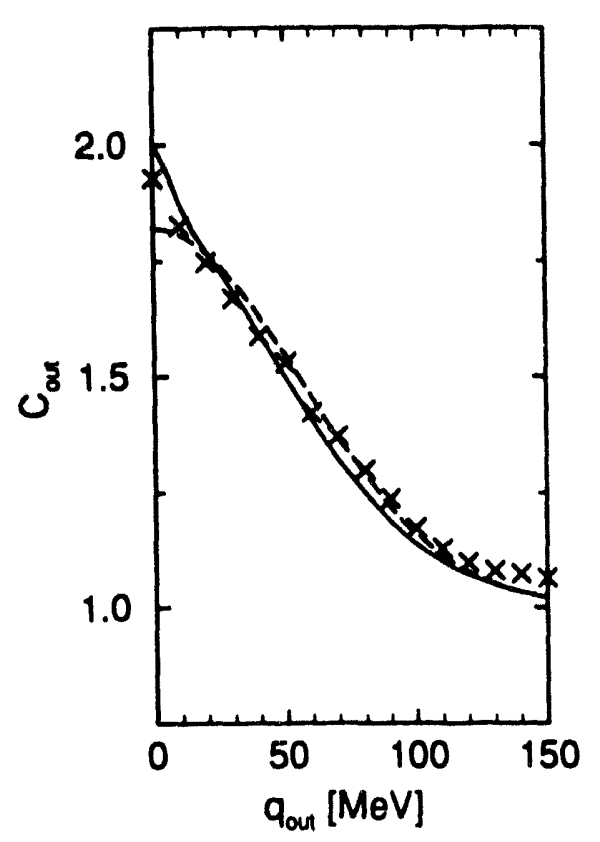

(a)

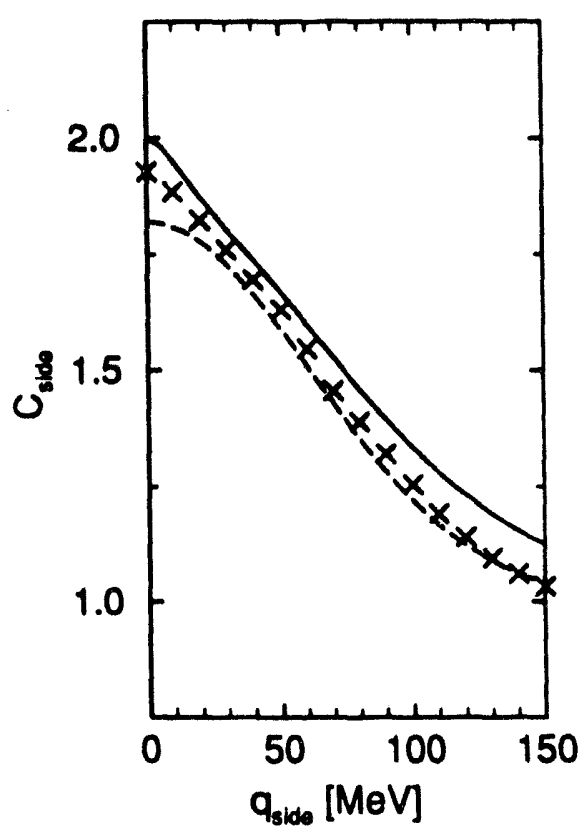

(b)

FIG. 6. Comparison between different methods to extract a correlation function from our simulation for the outward (a) and sideward (b) projection. The crosses are the results obtained by replacing the source function by a sum over delta functions given directly by the final distribution of momenta and last interaction points. The dashed line shows the result of a 3D Gaussian fit to these points. The full line is the correlation function calculated using the parameterization Eq. (A1). Note that the result from the parameterization goes to two for $q_{\text {out } / \text { side }}=0$ whereas the other methods show a smaller intercept due to finite binning over ranges of $y$ and $p_{T}$. 
TABLE III. Functions and parameters for the parameterization of the source function. Units are fermi for lengths and fermi/c for times.

\begin{tabular}{|c|c|c|c|}
\hline $\mathrm{i}$ & 1 & 2 & 3 \\
\hline$n_{i}$ & 128 & 15 & 11 \\
\hline$g_{i}^{\tau}$ & $a_{1}^{\tau} \exp \left(\frac{\left(\tau-b_{1}^{\tau}\right)^{2}}{2 c_{1}^{\tau^{2}}}\right)$ & \multicolumn{2}{|c|}{$a_{i}^{\tau} \exp \left(-\tau / b_{i}^{\tau}\right)$} \\
\hline & $a_{1}^{\tau}=0.18, b_{1}^{\tau}=5.0, c_{1}^{\tau}=2.2$ & $a_{2}^{\tau}=1.3, b_{2}^{\tau}=5.6$ & $a_{3}^{\tau}=0.11, b_{4}^{\tau}=22$ \\
\hline \multirow[t]{2}{*}{$g_{i}^{r}$} & $a_{i}^{r} r_{\perp} \exp$ & $\left.-\frac{\left(r_{1}-b_{i}^{r}\right)^{2}}{2 c_{i}^{2}}\right)$ & $a_{3}^{r} r_{\perp} \exp \left(-r_{\perp} / b_{3}^{r}\right)$ \\
\hline & $a_{1}^{r}=0.20, b_{1}^{r}=0.59, c_{1}^{r}=1.84$ & $a_{2}^{r}=0.043, b_{2}^{r}=0.010, c_{2}^{r}=4.8$ & $a_{3}^{r}=0.010, b_{3}^{r}=9.9$ \\
\hline \multirow[t]{2}{*}{$g_{i}^{\eta}$} & \multicolumn{3}{|c|}{$(\sqrt{2 \pi} \Delta \eta)^{-1} \exp \left(-\frac{\eta^{2}}{2 \Delta \eta_{i}^{2}}\right)$} \\
\hline & $\Delta \eta_{1}=0.47$ & $\Delta \eta_{2}=0.31$ & $\Delta \eta_{3}=0.57$ \\
\hline \multirow[t]{2}{*}{$g_{i}^{\phi}$} & \multicolumn{3}{|c|}{$1+b_{i}^{\phi} \cos (\phi)+c_{i}^{\phi} \cos (2 \phi)$} \\
\hline & $b_{1}^{\phi}=1.2, c_{1}^{\phi}=0.41$ & $b_{2}^{\phi}=1.4, c_{2}^{\phi}=0.95$ & $b_{3}^{\phi}=0.95, c_{3}^{\phi}=0.13$ \\
\hline
\end{tabular}


[1] Proceedings of Quark Matter '93, Nucl. Phys. A566 (1994).

[2] G. Roland et al. (NA35), Nucl. Phys. A566 (1994) 527c.

[3] H. Beker et, al. (NA44), Nucl. Phys. A566 (1994) 115c.

[4] D. Boal, C.K. Gelbke and B. Jennings, Rev. Mod. Phys.62 (1990) 553.

[5] S. Pratt, Phys. Rev. D33 (1986) 1314.

[6] G.F. Bertsch, Nucl. Phys. A488 (1989) 173c.

[7] G. Welke, et al., contribution to winter workshop on nuclear dynamics, Wayne State Univ. preprint WSU-NP-94-1.

[8] J. P. Sullivan, M. Berenguer, B. V. Jacak, S. Pratt, M. Sarabura, J. Simon-Gillo, H. Sorge and H. van Hecke, Phys. Rev. Lett. 70 (1993) 3000.

[9] J.P. Sullivan, M. Berenguer, D.E. Fields, B.V. Jacak, M. Sarabura, J. Simon-Gillo, H. Sorge, H. van Hecke and S. Pratt, Nucl. Phys. A566 (1994) 531c.

[10] H. deVries, C.W. de Jager and C. de Vries, At. Data Nucl. Data Tables 36 (1987) 495.

[11] K. Kohlemainen and M. Gyulassy, Phys. Lett. B180 (1986) 203.

[12] R. Venugopalan, M. Prakash, M. Kataja and P. Ruuskanen, Nucl. Phys. A566 (1994) 473c.

[13] J.D. Bjorken, Phys. Rev. D27 (1983) 140.

[14] R. Singer et al., Phys. Lett. B60 (1976) 385.

[15] M. Schouten et al., Z. Phys. C9 (1981) 93.

[16] M. Aguilar-Benitez, Z. Phys. C50 (1991) 405.

[17] S. Padula and M. Gyulassy, Nucl. Phys. A544 (1992) 537c. 
[18] E. Schnedermann, J. Sollfrank and U. Heinz, Phys. Rev. C48 (1993) 2462.

[19] U. Goerlach for the HELIOS Collaboration, Nucl. Phys. A544 (1992) 109c.

[20] J. Baechler et al., (NA35), Phys. Rev. Lett. 72 (1994) 1419.

[21] G. Bertsch, M. Gong, L. McLerran, V. Ruuskanen and E. Sarkkinnen, Phys. Rev. D37 (1988) 1202.

[22] J. Goity, Phys. Lett. 319 (1993) 401.

[23] G.F. Bertsch, P. Danielewicz and M. Herrmann, Phys. Rev. C49 (1994) 442.

[24] S. Pratt, Phys. Rev. Lett. 53 (1984) 1219.

[25] A.N. Makhlin and Yu.M. Sinyukov, Z. Phys. C39 (1988) 69.

[26] H. Barz, G. Bertsch, B. Friman, H. Schulz and S. Boggs, Phys. Lett. B265 (1991) 219.

[27] G.F. Bertsch and S. DasGupta, Phys. Reports 160 (1988) 512.

[28] D. Roehrich et al. (NA35), Nucl. Phys. A566 (1994) 35c.

[29] H. Bøggild et al., Phys. Lett. B302 (1993) 510.

[30] A.H. Mueller and J. Qiu, Nucl. Phys. B268 (1986) 427.

[31] J.P. Blaizot and A.H. Mueller, Nucl. Phys. B289 (1987) 847.

[32] P. Seyboth et al. (NA35), Nucl. Phys. A544 (1992) 531c.

[33] F.R. Brown, F.P. Butler, H. Chen, N.H. Christ, Z. Dong, W. Schaffer, L.I. Unger and A. Vaccarino, Phys. Rev. Lett. 65 (1990) 2491.

[34] N.H. Christ, Nucl. Phys. A544 (1992) 81.

[35] K. Werner, private communication.

[36] K. Werner, Physics Reports 232 (1993) 87. 
[37] B.R. Schlei, U. Ornik, M. Plümer and R.M. Weiner, Phys. Lett. B293 (1992) 275.

[38] U. Mayer, E. Schnedermann and U. Heinz, Phys. Lett. B294 (1992) 69. 

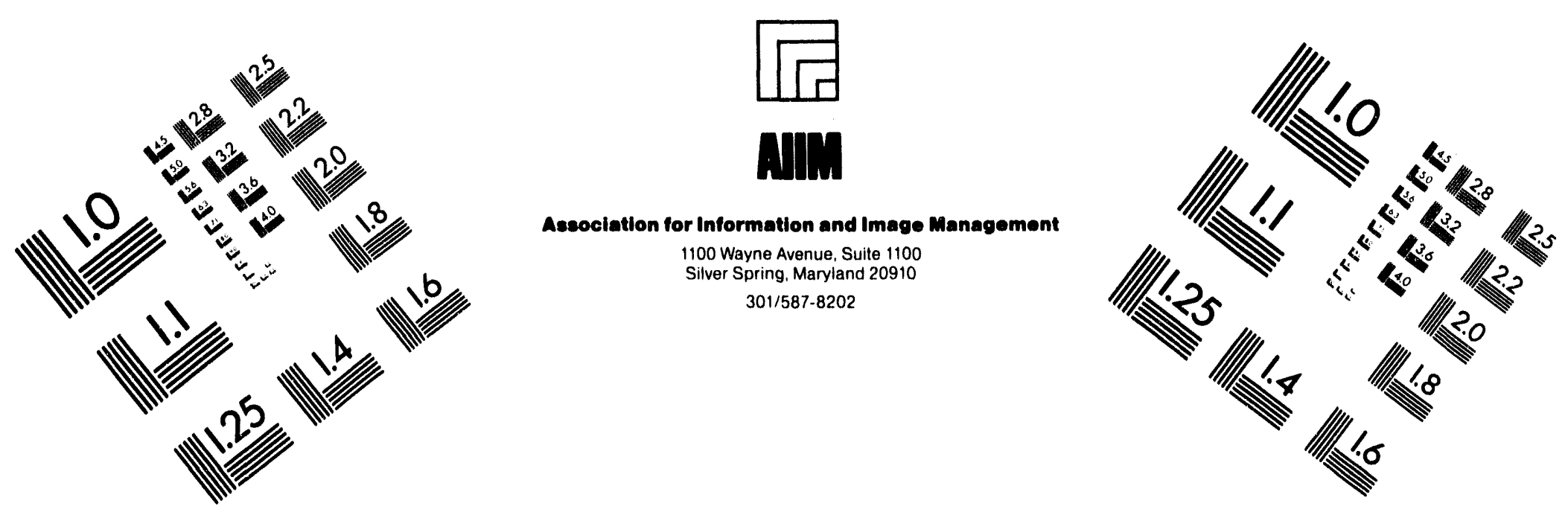

Centimeter

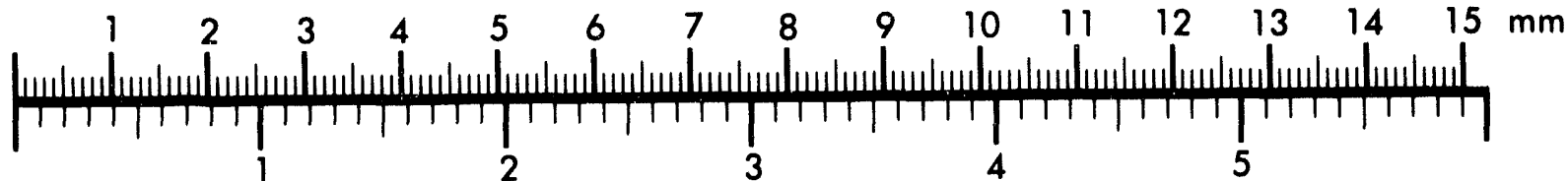
Inches

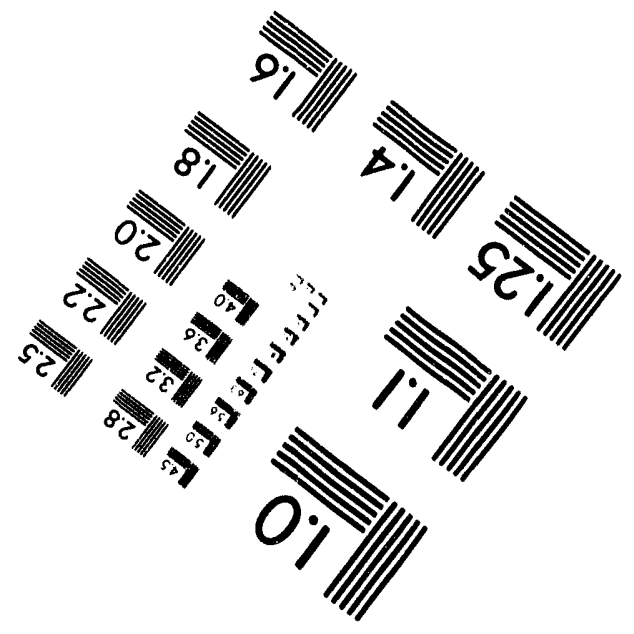

MANUFACTURED TO AIIM STANDARDS

BY APPLIED IMAGE, INC.

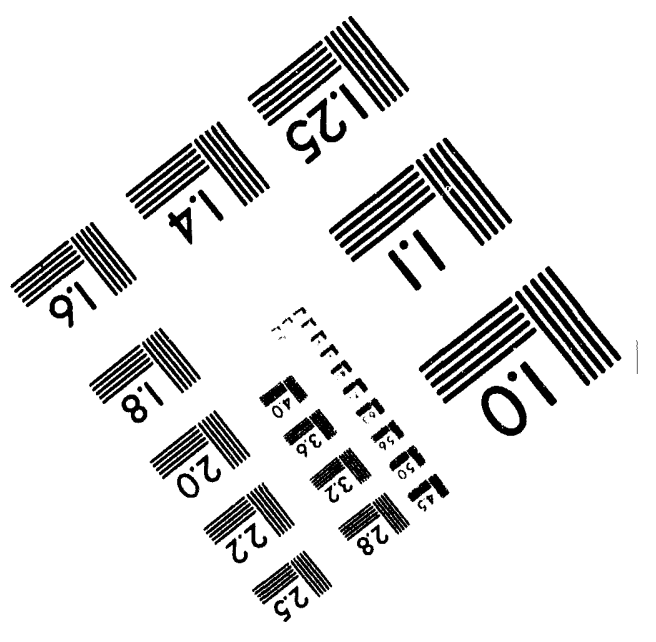




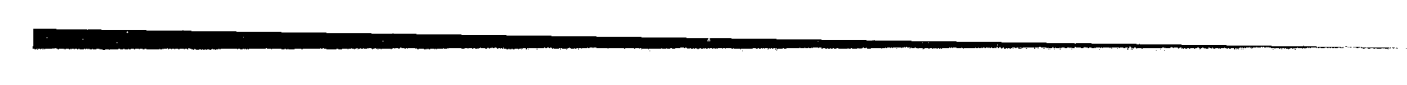



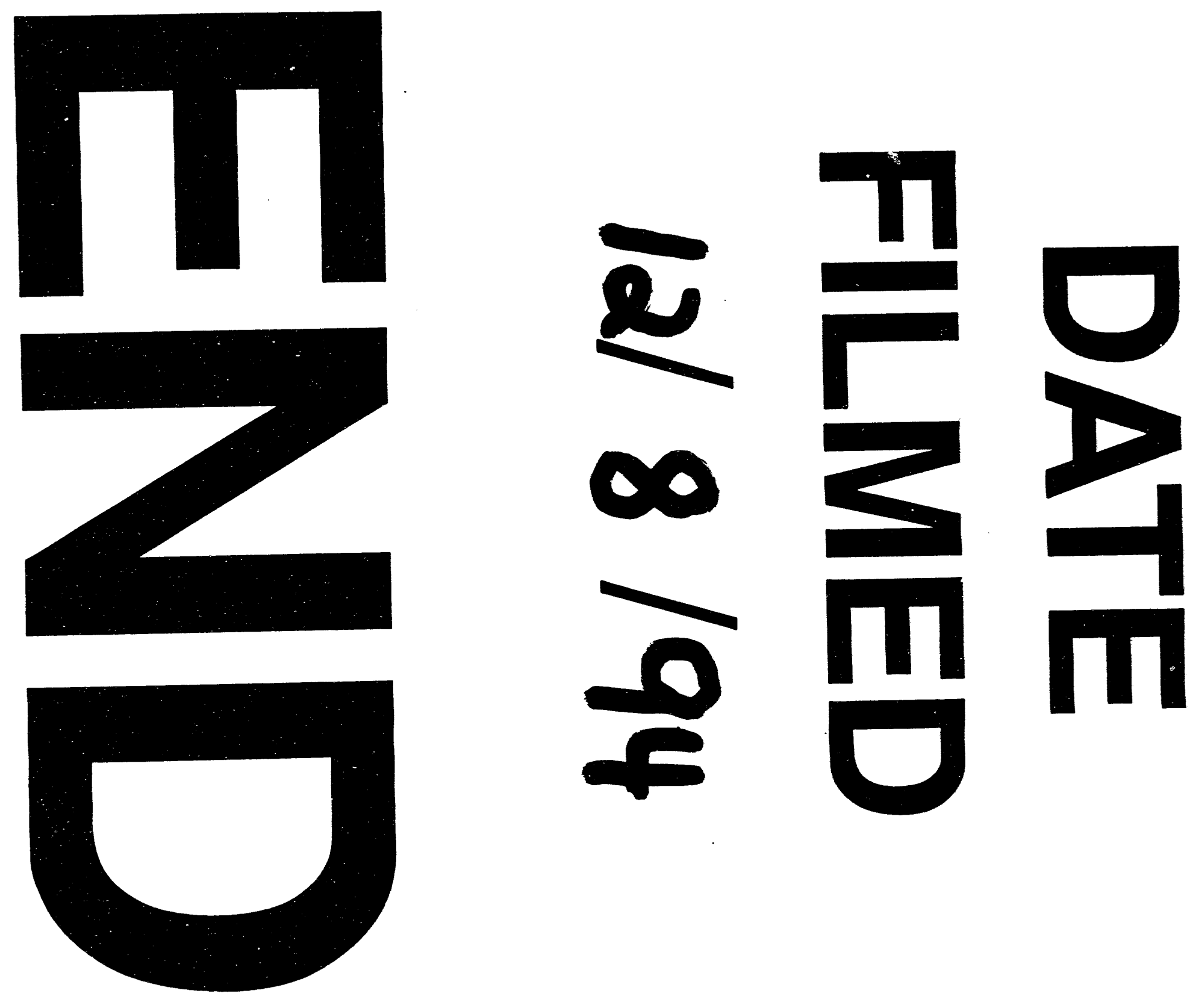